\title{
Review
}

Jinlong He, Zhen Cui and Yi Zhu*

\section{The role of caveolae in endothelial dysfunction}

https://doi.org/10.1515/mr-2021-0005

Received March 19, 2021; accepted August 3, 2021 ;

published online October 21, 2021

\begin{abstract}
Caveolae, the specialized cell-surface plasma membrane invaginations which are abundant in endothelial cells, play critical roles in regulating various cellular processes, including cholesterol homeostasis, nitric oxide production, and signal transduction. Endothelial caveolae serve as a membrane platform for compartmentalization, modulation, and integration of signal events associated with endothelial nitric oxide synthase, ATP synthase $\beta$, and integrins, which are involved in the regulation of endothelial dysfunction and related cardiovascular diseases, such as atherosclerosis and hypertension. Furthermore, these dynamic microdomains on cell membrane are modulated by various extracellular stimuli, including cholesterol and flow shear stress. In this brief review, we summarize the critical roles of caveolae in the orchestration of endothelial function based on recent findings as well as our work over the past two decades.
\end{abstract}

Keywords: atherosclerosis; caveolae; caveolin-1; endothelial cell; integrin; shear stress.

\section{Introduction}

Caveolae are 50-100 nm wide plasma membrane pits and are one of the most significant morphological characteristics of mammalian cells, rich in cholesterol, sphingolipids, and a complete family of membrane proteins called caveolin. They are a subset of lipid rafts because of their unique structure. Using transmission electron microscopy, caveolae were described as a bulb-shaped or cuplike

*Corresponding author: Yi Zhu, Department of Physiology and Pathophysiology, Tianjin Medical University, 22 Qixiangtai Rd, Tianjin 300070, China, E-mail: zhuyi@tmu.edu.cn. https://orcid.org/00000001-7725-9166

Jinlong He and Zhen Cui, Tianjin Key Laboratory of Metabolic Diseases, The Province and Ministry Co-sponsored Collaborative Innovation Center for Medical Epigenetics and Department of Physiology and Pathophysiology, Tianjin Medical University, Tianjin 300070, China uncoated invagination, which is different from other membrane subdomains such as clathrin-coated pits [1]. The lipid components of caveolae consist of cholesterol, sphingomyelin, glycosphingolipids, and saturated fatty acids. The protein components of caveolae, including caveolins, cavins, and pacsin/syndapin, are critical for their formation and function. Caveolin is a hairpin-like protein that is embedded in the plasma membrane, with both the N-terminal and C-terminal located in the cytoplasm. Caveolin acts as a partner and scaffold of signal molecules in caveolae and provides temporal and spatial regulation of signal transduction. There are three isoforms of caveolins in mammalian cells: caveolin-1, caveolin-2, and caveolin-3. Caveolin-1 and caveolin-2 are co-expressed ubiquitously in mammals, whereas caveolin-3 is expressed primarily in skeletal and cardiac myocytes. Caveolin-1, a $22 \mathrm{kDa}$ protein encoded by the CAV1 gene, is a major component of caveolae membranes in vivo and has been widely investigated. There are a quantal number of caveolin-1 molecules (144 \pm 39 ) incorporated in each caveolar coat [2]. Loss of caveolin-1 prevents caveolae formation, whereas caveolin-1 transfection in lymphocytes lacking both caveolae and caveolin expression promotes the formation of plasmalemmal caveolae [3]. Caveolin-1 binds to a variety of signaling molecules and receptors in endothelial cells (ECs), such as endothelial nitric oxide synthase (eNOS), G-protein, protein kinases, and growth factor receptors, and participates in a variety of cellular functions, including endocytosis, transcytosis, cholesterol transport, and signal transduction.

The density of caveolae depends on the cell type and conditions. In the kidney proximal tubule, caveolae and caveolin-1 are undetectable [1]. In contrast, caveolae are abundant in ECs, especially in the continuous endothelium, including that of the heart and skeletal muscle [4]. The caveolar density of ECs changes remarkably with time in culture. Although caveolae are still present in ECs immediately after isolation, their number is drastically reduced in culture [5]. The underlying reason might be that physiological shear stress is indispensable for maintaining a normal density of caveolae. In static cultured bovine aortic ECs (BAECs), the caveolar density is $(0.2 \pm 0.03)$ caveolae/ $\mu \mathrm{m}$ of plasma membrane [6]. However, after exposure to $10 \mathrm{dyn} / \mathrm{cm}^{2}$ laminar shear stress (LSS) in a

Ә Open Access. @ 2021 jinlong He et al., published by De Gruyter. (c) BY-Nc-ND This work is licensed under the Creative Commons Attribution-NonCommercialNoDerivatives 4.0 International License. 
parallel plate apparatus for $6 \mathrm{~h}$, the caveolae density at the plasma membrane increases six-folds [6].

Endothelial caveolae/caveolin-1 regulate various aspects of the cardiovascular system and have been associated with numerous cardiovascular diseases, including hypertension, atherosclerosis, angiogenesis, cardiac hypertrophy, pulmonary hypertension, and ischemic injury (Figure 1). Genetic ablation of caveolin-1 on an $\mathrm{ApoE}^{-/-}$background reduces the size of aortic plaques, despite the elevated non-HDL (high-density lipoprotein) plasma cholesterol levels [7]. Re-expression of caveolin-1 in ECs alone fully recovered aortic lesions in global $\mathrm{ApoE}^{-/-}$caveolin-1 ${ }^{-/-}$mice, indicating an atheroprone role of endothelial caveolin-1 [8]. A smaller number of caveolae is associated with hypertension. The density of caveolae in the ECs of aortas is markedly reduced in spontaneously hypertensive rats [9]. Caveolin-1 knockout mice fed a high-fat diet exhibit significantly increased systolic and diastolic blood pressure compared with those in wild-type mice, either on a normal chow diet or a highfat diet [10]. Caveolin-1 is also linked to the reninangiotensin-aldosterone system, which is involved in the pathogenesis of cardiovascular diseases, including hypertension, renal disease, and heart failure. Angiotensin II (Ang II) promotes association of Ang II type1 receptor (AT1R) with caveolin-1 and AT1R trafficking into lipid rafts in vascular smooth muscle cells (VSMCs) [11]. Arteries from caveolin-1 deficiency mice showed abnormalities in Ang II-induced contractile responses [12]. Among statin users, people carrying the caveolin-1 risk allele (rs926198), which is associated with decreased levels of caveolin-1, exhibited a $25 \%$ lower aldosterone level than those not carrying the caveolin-1 risk allele, indicating the association between caveolin-1 expression and aldosterone levels [13]. Furthermore, caveolin-1 deficiency causes accumulation of free cholesterol in mitochondrial membranes and alternation of mitochondrial metabolism, which might contribute to

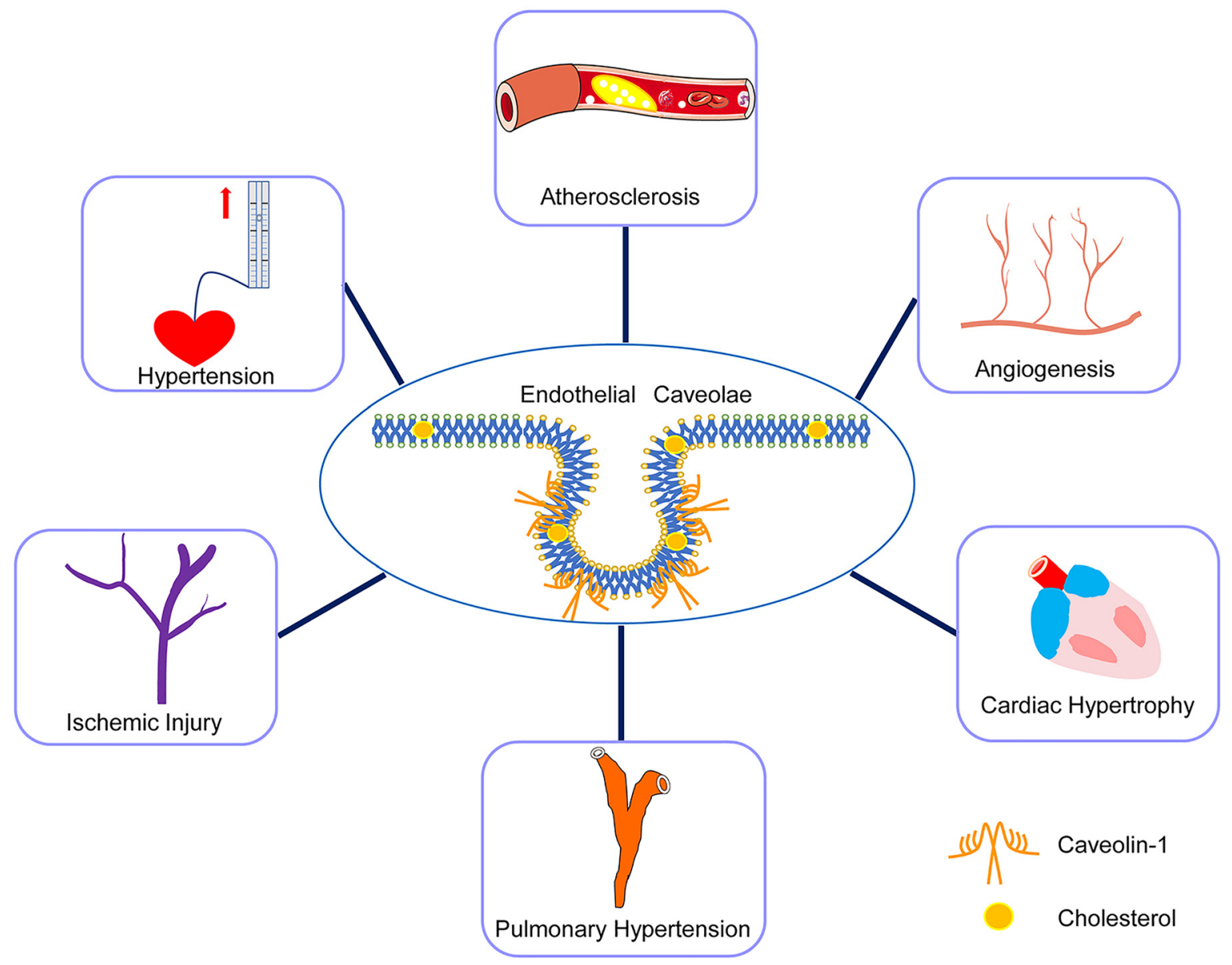

Figure 1: Endothelial caveolae/caveolin-1 and related cardiovascular diseases. Accumulating evidence indicates important roles of endothelial caveolae/caveolin-1 in cardiovascular diseases, including atherosclerosis, hypertension, ischemic injury, pulmonary hypertension, cardiac hypertrophy, and angiogenesis. 
mitochondrial related diseases [14, 15]. Importantly, caveolae serve as a platform to orchestrate endothelial functions, including cholesterol homeostasis, nitric oxide (NO) production, and mechano-transduction, and contribute to the progression of cardiovascular diseases. For example, statins modulate the translocation of proteins in lipid rafts. Atorvastatin treatment increases the localization of endoplasmic reticulum protein 46 in lipid rafts and hence inhibits reactive oxygen species production by nicotinamide adenine dinucleotide phosphate oxidase 2 (Nox2) in ECs [16]. Since the structure, formation, and general functions of caveolae have been well reviewed recently $[1,17]$, in this review, we will focus primarily on the role of caveolae in endothelial dysfunction and related cardiovascular diseases.

\section{Caveolae on endothelial dysfunction}

ECs lining the inner-most layer of all blood vessels are the main regulators of vascular wall homeostasis. Endothelial dysfunction, which is characterized by a shift in the actions of the endothelium toward reduced vasodilation and proinflammatory state, is the chief cause for the development of cardiovascular diseases, including hypertension and atherosclerosis. Endothelial dysfunction can be induced by a variety of noxious substances (e.g. oxidized cholesterol, constituents of cigarette smoke), hyperhomocysteinemia, hyperglycemia, and mechanical stress. Caveolae play critical roles in orchestrating endothelial functions, including cholesterol homeostasis and NO production, and serve as mechanosensors in ECs.

\section{Endothelial caveolae and cholesterol homeostasis}

Accumulation of cholesterol ester in the arterial wall promotes the formation of atherosclerotic plaque, and reverse cholesterol transportation is considered the main pathway by which accumulated cholesterol is transported from the vessel wall to the liver for disposal. Epidemiological evidence confirms the relationship between the levels of low-density lipoprotein (LDL) cholesterol and the risk of coronary artery diseases [18]. Cholesterol efflux, a critical part of the reverse cholesterol transportation, is a major process by which macrophages, ECs, and other cells within the vessel wall transfer the deposited cholesterol to apolipoprotein A-I (apoA-I) to form nascent HDL via
ATP-binding cassette family transporter (ABC) A1, ABC sub-family $G$ member 1 (ABCG1), and scavenger receptor class B type I (SR-B1) [19]. Receptors and transporters, including CD36, activin receptor-like kinase (ALK1), SR-B1, ABCA1, and ABCG1 are thought to localize in caveolae and mediate the uptake or efflux of cholesterol $[19,20]$.

LDL induces endothelial activation through multiple signaling pathways. The native LDL can be internalized via caveolin-1 and CD36 in caveolae, and LDL endocytosis is reduced in caveolin-1 deficient ECs [21]. Besides, LDL increases free cholesterol contents in the cell membrane, mainly in the caveolar fractions, in ECs in as early as 30 min [22]. Caveolin-1 directly binds cholesterol with high affinity with a 1:1 ratio. Cholesterol binds to both the C-terminal lipid-binding sites and scaffolding domain, which are critical for membrane attachment of caveolin-1 [23]. Hence, in ECs, caveolin-1 translocation from the cytosol to the cell membrane caveolae as well as the binding of caveolin-1 and caveolae-related signaling molecule Ras could be induced by LDL [22]. ABCA1, a crucial factor in maintaining sterol and lipid homeostasis by transporting cholesterol efflux to apoA-I, is also induced by LDL and cholesterol in ECs [24]. LDL transcriptionally regulates ABCA1 by activating liver $\mathrm{X}$ receptor/retinoid $\mathrm{X}$ receptor (LXR/RXR), and overexpression of ABCA1 can lower cellular cholesterol content and increase apoA-I-mediated cholesterol efflux in ECs [24, 25]. Besides, the atheroprotective flow pattern boosts the expression of both $\mathrm{ABCA} 1$ and $\mathrm{ABCG} 1$ by increasing the expression and activation of LXRs in ECs [26]. Using isolated intima of the thoracic aorta and aortic arch of C57/BL6 mice, our group found that the mRNA levels of LXR $\alpha$ and LXR target genes, including ApoE, ABCA1, ABCG1, and lipoprotein lipase, were higher in the thoracic aorta than in the aortic arch [26], thus highlighting the active participation of ABCA1/ ABCG1 in EC activation and atherosclerosis. ABCG1 deficiency in LDLR knockout (LDLR ${ }^{-/}$) mice increases early atherosclerotic lesion size but reduces advanced lesion size [27], possibly due to the diverse roles of ABCG1 in different cell types. Using ECs isolated from ABCG1-deficient mice, Whetzel et al. found ABCG1 deficiency reduced cholesterol efflux to HDL and activated endothelial interleukin(IL)-6-signal transducer and activator of transcription 3 (STAT3) signaling, thereby increasing monocyte adhesion to ECs and promoting EC dysfunction [28]. Found ABCG1 deficiency reduced cholesterol efflux to HDL and activated endothelial interleukin(IL)-6-signal transducer and activator of transcription 3(STAT3) signaling, thereby increasing monocyte adhesion to ECs and promoting EC dysfunction. However, local and vascular-specific gene transfer by adenoviral delivery of human ABCG1 in rabbits markedly blunted vascular inflammation and reduced atheroprogression [29]. Consistently, we 
constructed Tie2Cre-mediated conditional overexpression of ABCG1 in $\mathrm{LDLR}^{-/-}$mice and further demonstrated the atheroprotective role of ABCG1. Tie2Cre-mediated ABCG1 overexpression alleviated Western diet-induced atherosclerotic lesion formation in mouse aortas. ABCG1 overexpression in ECs reversed endothelial activation induced by cholesterol or oscillatory shear stress (OSS) by blocking cholesterol-induced endothelial activation of nuclear factor kappa B (NF-kB) and NLR family pyrin domain-containing protein 3 (NLRP3) inflammasome [30]. Hence, lipid-associated transporters localized in caveolae, including ABCA1 and ABCG1, prevent ECs from accumulating lipids by cholesterol efflux, and inhibit endothelial inflammatory activation induced by hyperlipidemia, inflammation, and disturbed flow (Figure 2).

Hypercholesterolemia promotes adherence of circulating blood monocytes to the intact intima, mainly mediated by the expression of cell adhesion molecules (CAMs), such as intercellular adhesion molecule-1 (ICAM-1) and vascular cell adhesion molecule-1 (VCAM-1) in the endothelium [31]. CAMs are distributed in membrane caveolae in ECs [32, 33]. Shortterm cholesterol exposure enhances lipopolysaccharide (LPS)-induced monocyte adhesion to ECs by reducing the caveolae localization of CAMs. Mechanistically, cholesterol loading in ECs decreases the association of ICAM-1 with caveolin-1 in caveolae [33]. In a hypercholesterolemia model using $\mathrm{ApoE}^{-/-}$mice, co-localization of ICAM-1 and caveolin-1 in aortic ECs was reduced after intraperitoneal LPS injection [33]. The translocation of CAMs induced by cholesterol may contribute to the synergistic effects of hypercholesterolemia and inflammation in atherogenesis.

\section{Endothelial caveolae and NO production}

The balance between endothelium-derived relaxing factors and endothelium-derived contracting factors is a hallmark of the physiological endothelium. Endothelial caveolae and caveolin-1 are indispensable for maintaining endotheliumdependent relaxation and myogenic tone in arteries by controlling eNOS activity and subsequent NO production.

NO, which acts as an endothelium-derived relaxing factor, is released from the endothelium driven by eNOS, which is activated by shear stress and agonists such as bradykinin and acetylcholine [34]. eNOS, which has been extensively studied for its pivotal role in vascular homeostasis, is a dually acylated peripheral membrane protein that targets the Golgi region and caveolae in ECs [35]. Besides NO production, eNOS might involve in cardiovascular diseases through its competing endogenous RNAs (ceRNA) including insulin receptor substrate 2 (IRS2) [36]. eNOS deficiency in $\mathrm{ApoE}^{-/-}$mice significantly increased blood pressure and promoted atherosclerotic lesion formation [37]. In addition, eNOS deficiency accelerated the formation of neointima and aortic aneurysms in $\mathrm{ApoE}^{-/-}$mice $[38,39]$. Transgenic overexpression of eNOS in $\mathrm{ApoE}^{-/-}$mice increased NO production in the aorta, but accelerated high-cholesterol dietinduced atherosclerosis [40]. Together, these findings provide important insights into the role of eNOS in the homeostasis of ECs and the mechanisms underlying endothelial dysfunction and vascular diseases.

eNOS localizes to the plasma membrane caveolae under normal conditions, and localization of eNOS within

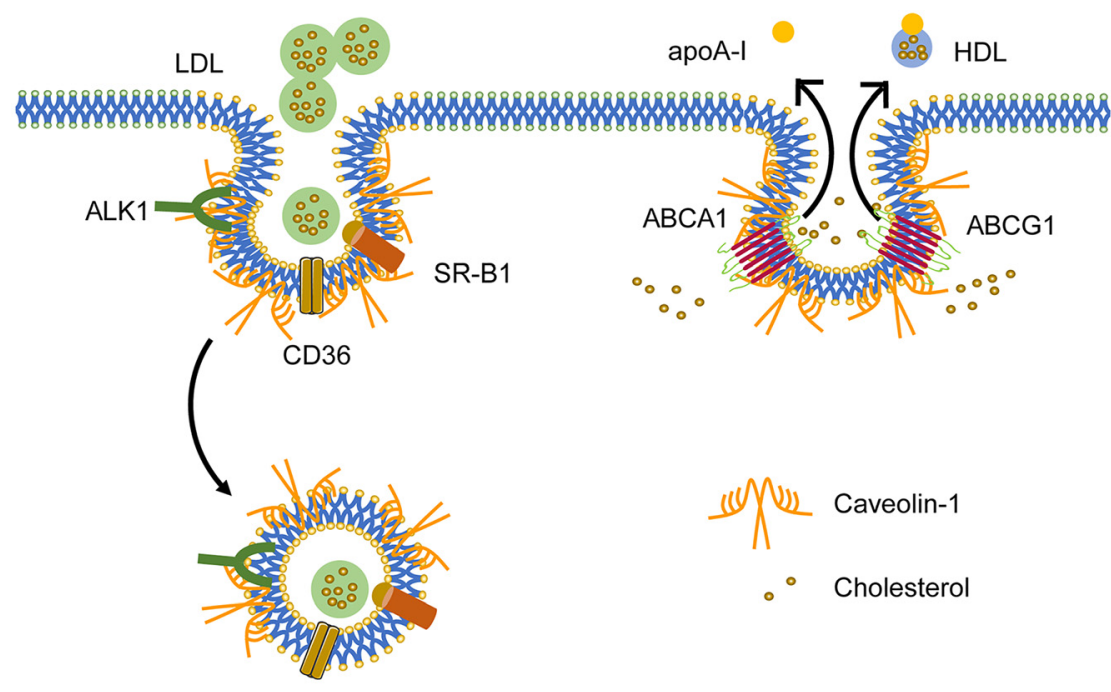

Figure 2: Endothelial caveolae mediate LDL endocytosis and cholesterol efflux. CD36, ALK1, SR-B1, ABCA1, and ABCG1 are localized in endothelial caveolae. CD36, ALK1, and SR-B1 mediate LDL endocytosis. ABCA1 and $A B C G 1$ facilitate the efflux of cholesterol to apoA-I and HDL, respectively. ABCA1: ATP-binding cassette family transporter $(A B C) A 1 ; A B C G 1$ : $A B C$ sub-family $G$ member 1; ALK1: Activin receptor-like kinase 1; HDL: High-density lipoprotein; LDL: Low-density lipoprotein; SR-B1: Scavenger receptor class B type I. 
the subcellular compartments determines and regulates its activity (Figure 3). When caveolae were disrupted by the membrane cholesterol-depleting agents, there was a decrease of NO production and NO-mediated relaxation in rat arteries [41, 42]. Mechanistically, caveolin-1 and heat shock protein 90 (Hsp90) could co-precipitate with eNOS from EC lysates respectively [43]. The association between eNOS and caveolin-1 in ECs is completely and specifically blocked by an oligopeptide within the caveolin sequence (amino acids 82-101), and the inhibition is competitively and completely reversed by $\mathrm{Ca}^{2+}$-calmodulin [44]. The presence of Hsp90 facilitates calmodulin dissociation of the eNOS-caveolin complex. The calcium-activated calmodulin recruits Hsp90 to the complex and facilitates the release of the caveolin inhibitory clamp, thereby resulting in the binding of Hsp90 to eNOS in a calmodulin-dependent manner and reducing the inhibitory actions of the caveolin-1 scaffolding peptide on eNOS activity [43]. Shear stress has been demonstrated to regulate eNOS at both transcriptional and post-transcriptional levels. $\mathrm{Ca}^{2+} /$ calmodulin-dependent protein kinase kinase (CaMKK) $\beta$, one of the major kinases activated by elevated intracellular calcium, is activated under pulsatile shear stress (PSS). CaMKK $\beta$ phosphorylated SIRT1 at Ser27 and Ser47 and enhanced SIRT1 stability in ECs, which contributed to eNOS phosphorylation at Ser1177 induced by PSS [45]. CaMKK $\beta$ deficiency blunted the phosphorylation of eNOS, induced EC activation, and accelerated atherosclerosis in mice [45]. However, genetic ablation of eNOS does not influence the extent of atheroprotection of caveolin-1 deficiency in $\mathrm{LDLR}^{-/-}$mice, which indicated that endothelial eNOS activation and NO production might not be involved in the atheroprotective phenotype of caveolin-1 deficiency mice [46]. Other caveolar components affect NO production, although the detailed mechanism is largely unknown. Caveolin-2 regulates the functional activity and stability of eNOS in ECs and controls NO production, which is independent of caveolae [47]. Caveolae associated protein 2 (cavin-2) is required for the proliferation, migration, and invasion of ECs in vitro by controlling the activity of eNOS and subsequent production of NO [47].

Lipoproteins have potent effects on eNOS function in caveolae via their action on membrane cholesterol homeostasis. LDL exposure increases the abundance of eNOS in caveolae and its association with caveolin-1 that is dependent on the actin-based cytoskeleton [48]. Disrupting stress fiber formation with cyto $\mathrm{D}$, an actin polymerization inhibitor, blocked the caveolar translocation of eNOS induced by the atherogenic concentrations of LDL [48]. HDL maintains the lipid environment in caveolae and causes direct activation of eNOS via SR-B1-induced kinase signaling [49]. Oxidized low-density lipoprotein (oxLDL) impairs NO production in ECs, which frequently occurs in early hypercholesterolemia-induced vascular diseases. One of the underlying mechanisms is that oxLDL can displace eNOS from caveolae by binding with SR-B1, resulting in the disruption of eNOS activation [50].

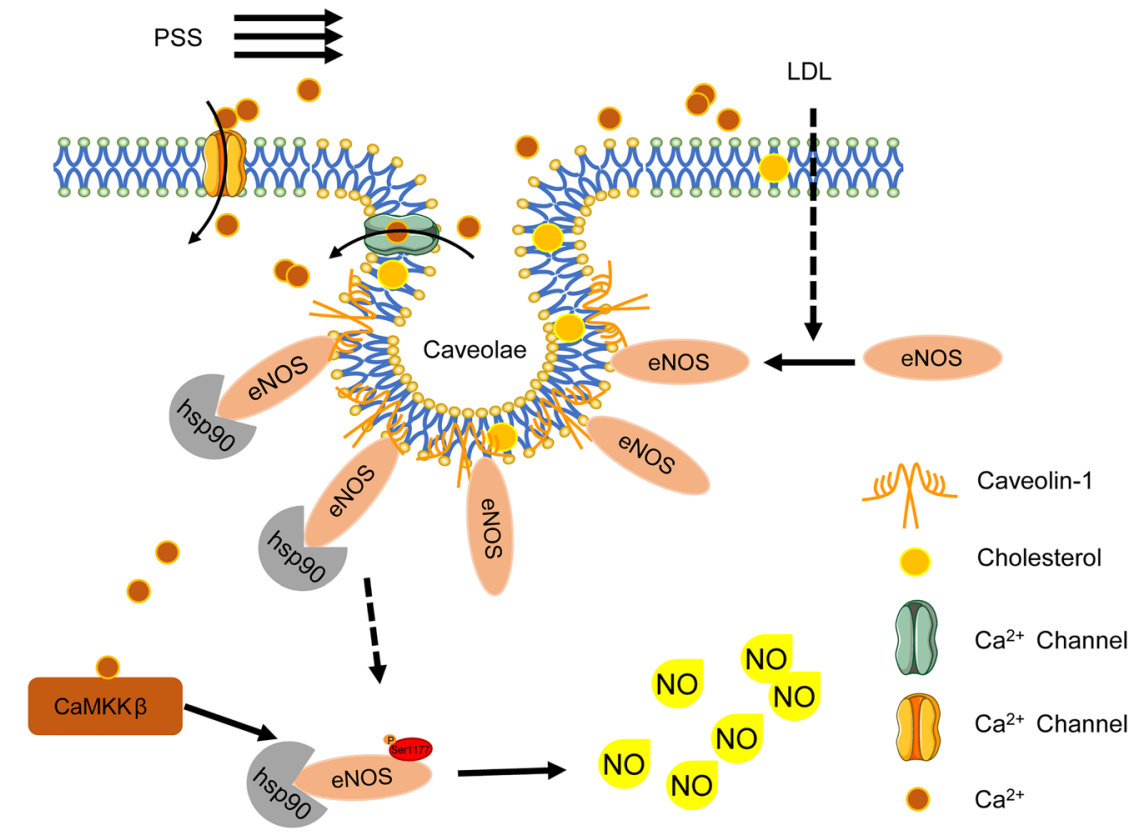

Figure 3: Endothelial caveolae regulate NO production. Binding with caveolin-1 causes inactivation of eNOS. PSS promotes eNOS phosphorylation at Ser1177 site and NO production in $\mathrm{ECs}$ via $\mathrm{Ca}^{2+} /$ calmodulindependent protein kinase kinase (CaMKK) $\beta$. LDL induces caveolar translocation of eNOS, which depends on the actin-based cytoskeleton. eNOS: endothelial nitric oxide synthase; LDL: Low-density lipoprotein; NO: Nitric oxide; PSS: Pulsatile shear stress. 


\section{Endothelial caveolae and mechanotransduction}

During endothelial dysfunction, the functions of the endothelium including permeability are markedly changed, leading to infiltration and upregulation of the expression of pro-inflammatory genes, such as monocyte chemoattractant protein-1, VCAM-1, ICAM-1, and E-selectin [51]. These changes preferentially occur along the arterial curvature or branching at sites that experience lower and multi-directional shear stress, which might be due to endothelial activation induced by shear stress. Typically, there are two major types of shear stress in vivo: disturbed flow and unidirectional laminar flow [52]. Unidirectional laminar flow is normally observed in the straight parts of the arteries, where blood flow is generally uniform and steady. Steady laminar flow with high shear stress elicits a quiescent anti-inflammatory and atheroprotective phenotype in ECs. In contrast, disturbed flow, which induces an atheroprone phenotype and oxidative stress in ECs, is usually observed in regions of arteries that curve sharply or in arteries with branching points and blood flow is unidirectional and irregular. By inducing mechanosensitive transcription factors, including Krüppel-like factor (KLF)2, KLF4, nuclear factor (erythroidderived 2)-like 2 (NRF2), NF-kB, activator protein 1 (AP-1), and hypoxia inducible factor 1 alpha (HIF-1 $\alpha$ ), disturbed flow and unidirectional laminar flow exert atheroprone and atheroprotective roles, respectively [53].

Moreover, shear stress involves in the control of EC cytoskeletal structure and function. Rac1 activation mediates cell alignment NF-kB-dependent expression of ICAM-1 induced by shear stress [54]. Cell cytoskeleton including microtubules, actin filaments, and intermediate filaments could associate with caveolae and is critical for the organization, trafficking, and recycling of caveolae [55]. Exposure to shear stress alters caveolae density and leads to enhanced mechanosensitivity to subsequent changes in hemodynamic forces. LSS enhances the caveolae density at the luminal plasma membrane of BAECs compared to static controls [6]. Hence, caveolae in ECs are involved in the regulation of vascular function by sensing or transducing hemodynamic changes into biochemical signals.

\section{Endothelial caveolae and ATP synthase $\beta$ chain (ATPS $\beta$ )}

Adenosine triphosphate (ATP) synthase, a membranebound complex with enzyme function and an iontransporter pump located in the inner mitochondrial membrane, is dedicated to the hydrolysis and synthesis of
ATP [56]. ATP synthase is composed of two main regions: the hydrophilic head $F_{1}$ domain and hydrophobic rotor part $\mathrm{F}_{0}$ domain; further, the peripheral rhizome is connected to it. The $F_{1}$ domain is located in the inner basement membrane of the mitochondria and consists of five subunits: $\alpha, \beta, \gamma, \delta$, and $\varepsilon$ [57]. The inhibition of ATP synthase with oligomycin or $\alpha$-ketoglutarate $(\alpha-K G)$ prolongs the lifespan of adult Caenorhabditis elegans [58]. In addition to being a major player in normal cellular energy supply, studies have shown that ATP synthase is involved in pathophysiology of several diseases. For example, ATP synthase is damaged by lipoxidation in human aging brains, which in turn affects cellular oxidative stress and damages neurons [59].

ATPS $\beta$ is a subunit of the ATP synthase $F_{1}$ hexamer that mainly plays a catalytic role and exists on the cell plasma membrane. It was originally thought that ATP synthase only exists on the inner mitochondrial membrane, until ATP synthase was detected in the K562 cells of chronic myeloid leukemia. ATP synthase can be used as a ligand to participate in the cytolytic pathway of naive natural killer (NK) and lymphokine-activated killer (LAK) cells in the effector stage and is called ectopic ATP synthase [60]. Since then, other studies have found the expression of ectopic ATP synthase in hepatocytes, keratinocytes, adipocytes, cancer cells, the central nervous system, and ECs. Ectopic ATP synthase can bind to angiostatin on EC and mediate its antiangiogenic effects and the downregulation of EC proliferation and migration [61]. ATP synthase on the surface of ECs binds to K1-5 and participates in the induction of caspase activation and apoptosis [62]. In addition, ATP synthase is a high-affinity receptor for lipid-free apoA-I and releases extracellular ADP in response to EC anti-apoptosis and proliferation [63].

Through analysis of matrix-assisted laser desorption ionization-time of flight mass spectrometry (MALDI-TOF MS) of caveolae-associated proteins affected by cholesterol loading in ECs, we found an increase in ATPS $\beta$ expression. Notably, the increase in ATPS $\beta$ in caveolae is due to its redistribution within the cell, while exhibiting a compensatory decrease in the mitochondria [64]. The glutathione S-transferase (GST)-pull-down experiment showed that ATPS $\beta$ and caveolin-1 physically bind to each other in ECs [64]. Consistently, Yamamoto et al. [65] showed that ATP synthase and caveolin-1 co-localized in lipid rafts of ECs and such localization can be eliminated by $m \beta$-CD. Disrupting the formation of actin polymers with cyto-B blocks the interaction between ATPS $\beta$ and caveolin-1 in ECs, suggesting that ATPS $\beta$ might be transported from the mitochondria to the plasma membrane through the cytoskeleton [64]. Shear stress induces 


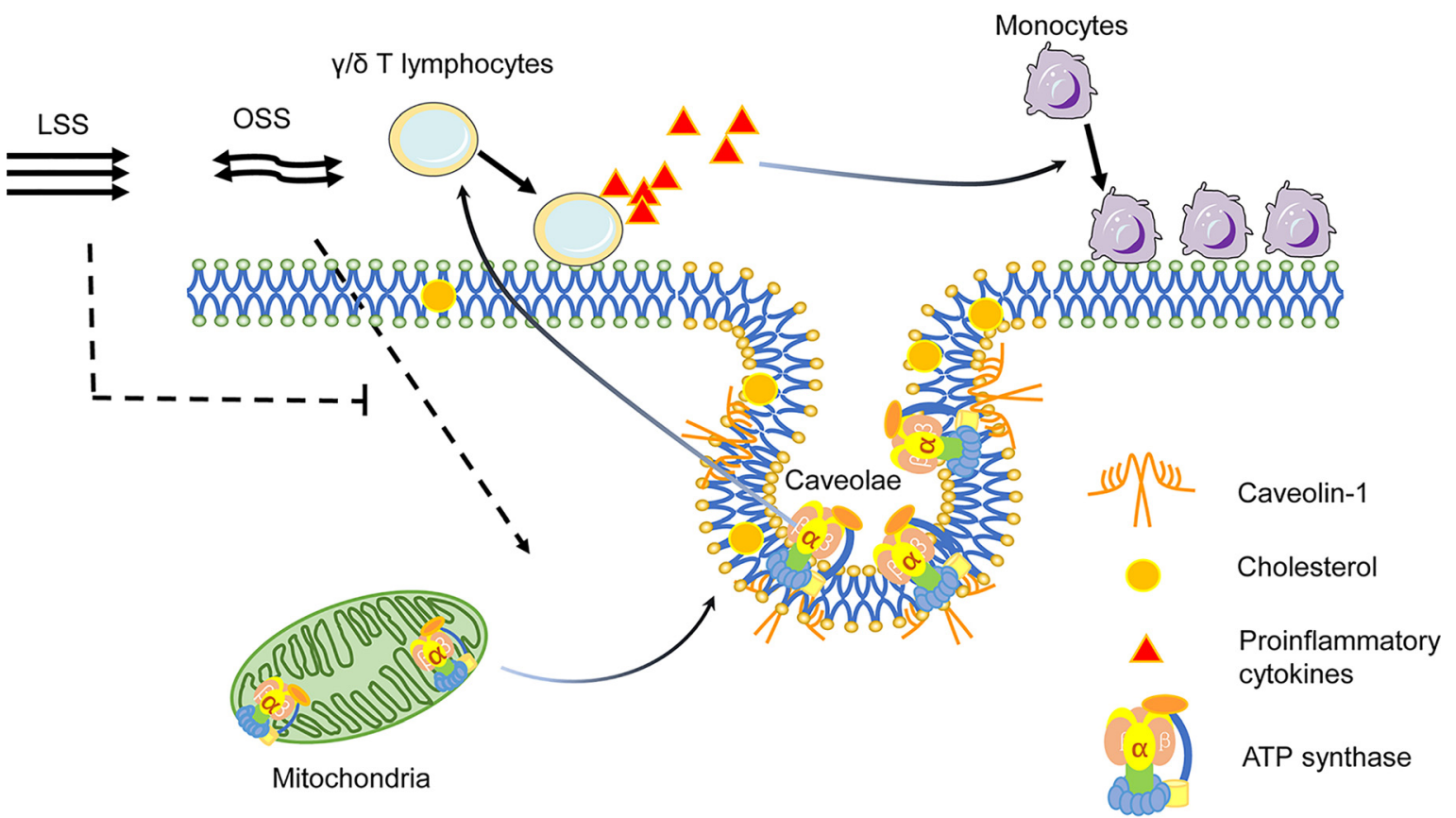

Figure 4: Shear stress-induced ATP synthase membrane translocation mediates the adhesion of inflammatory cells to ECs. LSS inhibits, while OSS promotes the translocation of mitochondrial ATPS $\beta$ to caveolae. Membrane ATPS $\beta$ facilitates the adhesion of $\gamma / \delta$ T cells, leads to the release of cytokines (IFN $\gamma$ and TNF $\alpha$ ) from the activated T cells and subsequent adhesion of monocytes to ECs. ATPS $\beta$ : ATP synthase $\beta$ subunit; IFN- $\gamma$ : Interferon gamma; LSS: Laminar shear stress; OSS: Oscillatory shear stress; TNF $\alpha$ : Tumor necrosis factor $\alpha$.

subcellular translocation of ATPS $\beta$ in ECs. Under the stimulation of LSS, ATPS $\beta$ translocates from the plasma membrane to the mitochondria and transiently increases the expression of ATPS $\beta$ in mitochondria. In contrast, OSS increased the expression of ATPS $\beta$ on the cell membrane but decreased its expression in mitochondria [66]. As the major component of the caveolae lipid core, the cholesterol content participates in the fluidity of the cell membrane and affects the membrane translocation of caveolin-1, which contributes to OSS-induced translocation of ATPS $\beta$ [66]. Both OSS and cholesterol treatment increased the adhesion of $y / \delta \mathrm{T}$ lymphocytes to ECs, leading to the release of cytokines (IFNy and tumor necrosis factor $\alpha[\mathrm{TNF} \alpha])$ from the attached $y / \delta \mathrm{T}$ cells, resulting in endothelial activation and atherogenesis [66] (Figure 4).

\section{Endothelial caveolae and Integrin-YAP}

Integrins are heterodimeric adhesion receptors comprised $\alpha$ and $\beta$ subunits, which are composed of a large extracellular region, a transmembrane region, and a relatively short cytoplasmic domain. The short cytoplasmic domain can interact with a variety of cytoskeleton proteins and intracellular signaling molecules to participate in a series of signal transduction pathways. Currently, $18 \alpha$ subunits and eight $\beta$ subunits have been identified in vertebrates that constitute at least 24 different integrins. The $\alpha$ subunit mainly determines the ligand type, and both the $\alpha$ and $\beta$ subunits are involved in cell signal transduction. Currently, 12 integrin heterodimers, namely $\alpha 1 \beta 1, \alpha 2 \beta 1, \alpha 3 \beta 1$, $\alpha 4 \beta 1, \alpha 5 \beta 1, \alpha 6 \beta 1, \alpha 6 \beta 4, \alpha 9 \beta 1, \alpha v \beta 1, \alpha v \beta 3, \alpha v \beta 5$, and $\alpha v \beta 6$, are known to play critical roles in ECs and are involved in the progression of cardiovascular diseases [67].

Arginine-glycine-aspartic acid (RGD)-containing proteins, such as fibronectin, fibrinogen, and thrombospondin-1, which are endogenous ligands of endothelial integrins, including $\alpha 5 \beta 1, \alpha v \beta 3$, and $\alpha v \beta 5$, are deposited in the matrix during the early stages of atherosclerosis [68]. In an atheroprone model with partial ligation of the left carotid artery in $\mathrm{ApoE}^{-/-}$mice, treatment with the $\alpha \mathrm{v}$ inhibitor $\mathrm{S} 247(40 \mathrm{mg} / \mathrm{kg}$ per day) via osmotic minipump for 7 days significantly reduced infiltration of macrophages in plaque. Meanwhile, S247 also attenuated endothelial activation evidenced by reduced VCAM-1 expression in the endothelium of the left carotid artery in mice [69]. ATN-161, a peptide mimetic of the Pro-His-Ser-Arg-Asn (PHSRN) sequence of fibronectin, inhibits $\alpha 5$ activation. Intraperitoneal administration of ATN-161 ( $5 \mathrm{mg} / \mathrm{kg}$, 3 times a week) for eight weeks signifcantly limited high-fat Western diet-induced atherosclerosis and reduced plaque sizes, without affecting the levels of total cholesterol, HDL cholesterol, LDL cholesterol, and 
triglycerides in $\mathrm{LDLR}^{-/-}$mice [70]. Consistently, vascular endothelial (VE)-cadherin Cre-induced-endothelial-specific knockout of $\alpha v$ reduced endothelial activation and the plaque area after partial carotid ligation in $\mathrm{ApoE}^{-/-}$mice [69]. Heterozygous knockout of $\alpha 5$ in $\mathrm{LDLR}^{-/-}$mice caused $\sim 50 \%$ decrease in the total lesion area in a Western diet-induced atherosclerosis model [71]. Integrin $\alpha 5 / 2$ chimera knock-in mice showed reduced shear-dependent inflammatory responses in the arteries [72]. Integrin $\alpha 5 / 2$ knock-in mice in $\mathrm{ApoE}^{-/-}$background showed smaller plaques in the aortic root than those of $\mathrm{ApoE}^{-/-}$mice, with reduced leukocyte content and metalloproteinase expression [73]. Interference with endothelial $\beta 1$-matrix adhesion by the monoclonal IgM Ha2/5 initiated F-actin conformational changes and increased the permeability of primary cerebral microvascular endothelial cell monolayers [74]. Endothelialspecific deletion of talin1 in established blood vessels reduced $\beta 1$ activation and disorganized adherens junctions, causing intestinal vascular hemorrhage and death. Treatment of talin-deficient ECs with $\beta 1$ activating antibody (9EG7) rescued the EC barrier function, indicating that talin-dependent activation of $\beta 1$ is required for maintaining VE-cadherin organization and EC barrier function [75]. Mutation of the integrin-binding site in phosphodiesterase 4D5 (PDE4D5) reduced atherosclerotic lesion size and inflammatory status in hyperlipidemic mice and enhanced plaque stability [76].

Integrin-mediated adhesion maintains membrane domains at the plasma membrane. Electron microscopy revealed that the number of caveolae in the plasma membrane decreased after detachment ( 47.5 caveolae per $\mu \mathrm{m}$ of membrane at $30 \mathrm{~s}$ compared to 12.9 at $2 \mathrm{~h}$ after detachment), indicating internalization of caveolae [77]. Disrupted caveolae by depleting membrane cholesterol with $\mathrm{m} \beta-\mathrm{CD}$ mimicked the loss of adhesion and prevented Rac1 translocation to the cell membrane [78]. Meanwhile, caveolae trafficking is involved in signal transduction of integrins. Soft substrates markedly enhanced the internalization of integrins through caveolae/raft-dependent endocytosis. Caveolae-mediated $\beta 1$ internalization in bone marrow mesenchymal stem cells contributes to soft substratetriggered neurogenic fate determination through inhibition of the bone morphogenetic proteins (BMP)/ small mothers against decapentaplegic (Smad) signal pathway [79]. Integrins in ECs are shear stress-sensitive, and their activation is essential for transmitting mechanical stimuli to the intracellular biochemical pathways. Emerging evidence indicates that caveolae, membrane receptors, cytoskeletal proteins, and extracellular matrixes are linked to integrin activation in the context of mechanotransduction.
Extracellular matrix (ECM) proteins, including collagens, laminins, fibronectin, fibrinogen, and vitronectin, can bind to endothelial integrin and promote its activation [68]. Fibronectin mediates $\alpha 5 \beta 1$ activation in ECs and promotes endothelial activation [71, 80]. ECM may also antagonize the activation of integrins. Our group recently revealed that cartilage oligomeric matrix protein (COMP) directly binds to $\alpha 5$ via its $C$-terminus and inhibits both disturbed flow-induced and fibronectin-induced $\alpha 5$ activation by inducing a more bent conformation of $\alpha 5 \beta 1$ in ECs [81]. COMP is a $524 \mathrm{kDa}$ pentameric non-collagenous matricellular glycoprotein, predominantly found in cartilage and tendon [82]. OSS induced $\alpha 5$ activation in human umbilical vein endothelial cells (HUVECs), which was inhibited by COMP, but not other ECMs, including CCN1, CCN3, and thrombospondin 1 . Consistently, $\alpha 5$ activation was aggravated in the inner curvature of the aortic arch compared with that in the thoracic aorta, which was further enhanced in $\mathrm{COMP}^{-/-}$mice compared with wild-type mice. The OSS-induced and fibronectin-induced phosphorylation of focal adhesion kinase (FAK) and Src, which are downstream signals of $\alpha 5$, were also blocked by COMP [81]. A peptide of COMP (C-terminus, amino acid 531-554) mimics the protective role of COMP in blocking OSS-induced $\alpha 5$ activation. Furthermore, the peptide retarded EC activation and atherosclerosis plaque formation in $\mathrm{ApoE}^{-/-}$mice [81]. In addition, COMP binds directly to the $\beta$-tail domain of $\beta 3$ via its $\mathrm{C}$-terminus in macrophages and binds directly to $\alpha 7$ in VSMCs $[83,84]$. Blocking of the binding of COMP and $\beta 3$ by the $\beta$-tail domain mimicked the COMP deficiency-induced shift in macrophage phenotypes [83].

Membrane translocation of integrin to endothelial caveolae is critical for its activation induced by shear stress. Like ATPS $\beta, \alpha 5$ membrane translocation into caveolae could also be triggered by shear stress. Quantitative proteomic analysis of lipid rafts from ECs exposed to OSS or PSS revealed that $\alpha 5$ was most remarkably elevated in lipid rafts under OSS among the 396 proteins redistributed in lipid rafts. Consistently, en face immunostaining revealed enhanced activated $\alpha 5$ staining and its colocalization with flotillin-2, a lipid raft marker, in atheroprone areas (aortic arch) in comparison with the atheroprotective area (thoracic aorta), despite no difference in total $\alpha 5$ expression [71]. Shear stress also triggers $\beta 1$ translocation into plasmalemmal caveolae by enhancing the formation of the $\beta 1 /$ phosphotyrosine caveolin-1 signaling complex in ECs [85]. These OSS-imposed effects, including the increase of membrane cholesterol and $\alpha 5$ activation, disappeared in ECs pretreated with $\mathrm{m} \beta$-CD to deplete cholesterol, which was restored when supplemented with cholesterol. Similarly, knockdown of 
caveolin-1 in ECs disrupted the structure of caveolae and prevented OSS-induced $\alpha 5$ membrane translocation and activation [71].

The membrane translocation of integrins to caveolae induced by shear stress is related to membrane fluidity and integrin carriers. OSS increased the time-resolved fluorescence anisotropy of 1, 6-diphenyl-1, 3, 5-hex-atriene (DPH) in ECs, indicating a decrease in membrane fluidity [71]. In contrast, PSS increased membrane fluidity and attenuated cholesterol content, which was abolished by cholesterol supplementation [71]. Similarly, cholesterol supplementation to adult mesenchymal stem cells, which leads to an increase in membrane cholesterol and decrease in membrane fluidity, increased the expression of $\alpha 1, \alpha 4$, and $\beta 1$ on the cell surface and adhesion rates of cells to fibronectin and collagen [86]. Cholesterol content and membrane fluidity regulated ionizing radiation-induced $\beta 4$ phosphorylation and cellular senescence in cancer cells [87].

Recently, our group identified a novel mechanism of OSS-induced $\alpha 5$ membrane translocation with an integrin carrier, annexin A2. Annexin A2, a member of the annexin family, is a $\mathrm{Ca}^{2+}$-dependent phospholipid-binding protein [88]. It consists of a disordered $\mathrm{N}$-terminus, followed by a conserved C-terminal CTD comprising four repeats. Specifically, the $\mathrm{C}$ terminal consists of four homologous repeats, each of which contains five $\alpha$-helix structures, forming a conserved disk capable of binding calcium ions and regulating the binding of annexin A2 to phospholipid molecules $[89,90]$. The N-terminal is the functional domain, which has binding activity and contains P11, tPA binding sites, Tyr23, Ser11, Ser25 phosphorylation sites, and a nuclear output signal $[90,91]$. Using functional proteomics, annexin A2 was found to be significantly decreased in caveolae-enriched fractions of ECs after cholesterol exposure for $4 \mathrm{~h}$ [64]. Annexin A2 is an important factor in the experimental hepatopulmonary syndrome serum-induced proliferation of pulmonary arterial smooth muscle cells. Upon hepatopulmonary syndrome serum stimulation, annexin A2 translocated to the caveolae, which was inhibited by the application of $\mathrm{m} \beta$-CD. Conversely, overexpression of caveolin-1 resulted in the relocation of annexin A2 from caveolae and negatively regulated extracellular signal-regulated kinase (ERK)1/2 and NF-kB activation, which inhibited the annexin A2-modulated proliferative behavior of the pulmonary arterial smooth muscle cell [92]. In addition, tumor cells incubated with LGRFYAASG-pen (an annexin A2-targeting peptide) showed disruption of filamentous actin, focal adhesions, and caveolae-mediated membrane trafficking, resulting in impaired cell adhesion and migration [93]. There was an increase in the proportion of annexin A2 along with $\alpha 5$ in lipid raft fractions in HUVECs treated with OSS, whereas there was a decrease in the protein level of annexin A2 in non-lipid rafts. The activation of $\alpha 5$ and markers of endothelial activation induced by OSS were abolished by annexin A2 knockdown. A direct interaction between annexin $\mathrm{A} 2$ and the $\alpha 5$ cytoplasmic domain was demonstrated in ECs [94]. OSS increased the binding of annexin $\mathrm{A} 2$ and $\alpha 5$, thus promoting the translocation of $\alpha 5$ into lipid rafts and facilitated integrin activation [94]. Hence, annexin A2 serves as an integrin carrier to mediate a5 membrane translocation under shear stress.

Shear stress-induced $\alpha 5$ activation promotes endothelial activation and atherosclerosis by activating integrin downstream signaling pathways such as FAK, NLRP3 inflammasome, PDE4D5, and yes-associated protein (YAP)/ and transcriptional co-activator with PDZ-binding motif (TAZ). FAK, a key regulator of cell adhesion and migration, mediates shear stress-induced integrin activation. Interaction of the $\beta$ subunit of integrins and FAK causes kinase autophosphorylation at Y397 of FAK [95]. Consistent with $\alpha 5$ activation, FAK phosphorylation was induced by both oxLDL and OSS [70, 81]. The $\alpha 5 \beta 1$-blocking antibodies P1D6 and SNAKA52, but not the $\alpha v \beta 3$-blocking antibody LM609 and $\alpha v \beta 5$-blocking antibody P1F6, inhibited FAK phosphorylation induced by oxLDL [70]. FAK knockdown with siRNA or inhibition with PF-573228 inhibits oxLDL-induced NF- $k B$ activation and VCAM-1 expression in ECs [70]. COMP acts as an $\alpha 5$ antagonist and inhibits both OSS-induced and fibronectin-induced FAK phosphorylation in ECs, whereas COMP knockout aggravated FAK phosphorylation in the aortic arch of mice [81]. NLRP3 inflammasome activation triggers the cleavage of pro-IL-1 $\beta$ and pro-IL-18 and secretes mature forms of these mediators to promote further inflammatory processes. $\alpha 5$ neutralizing antibody or caveolin-1 deficiency inhibits OSS-induced cleavage of procaspase- 1 and pro-IL-1 $\beta$ in ECs [71]. $\alpha 5$ directly binds to PDE4D5, which induces protein phosphatase 2A (PP2A)-dependent dephosphorylation of PDE4D5 at the inhibitory site Ser651 [72]. The PDE4 inhibitor rolipram abolishes flow-dependent NF- $\mathrm{KB}$ activation in ECs, and endothelial-specific knockdown of PDE4D attenuates flowdependent endothelial activation in mice [72].

Emerging evidence demonstrates that YAP/TAZ, the major downstream effectors of the Hippo pathway, mediate endothelial activation induced by integrins in the context of mechanotransduction (Figure 5). The core components of the Hippo pathway in mammals contain kinase cascade of mammalian Ste20-like kinases 1/2 and large tumor suppressor $1 / 2$, and controls organ size by regulating cell survival, proliferation, and apoptosis [96]. When the Hippo signaling pathway is inactivated, YAP and its paralog TAZ 
translocate into the nucleus and activate gene transcription by binding to transcription factors [96]. The subcellular localization of YAP, which is important for its transcriptional activity, is regulated by its phosphorylation status [96]. Negative feedback regulation exists between YAP/TAZ and components of caveolae. Caveolin-1 serves as a negative regulator of YAP/TAZ [97, 98]. Lowering either caveolin-1 or cavin-1 expression caused increased the expression of YAP targeted genes [97, 98]. Genetic deletion of caveolin-1 and caveolin-3 in zebrafish induce a clear increase in the YAP nuclear-to-cytoplasmic ratio. Conversely, YAP/TAZ could bind with transcriptional enhanced associate domains (TEADs) and transcriptionally regulates the expression of caveolin-1 and cavin-1. YAP/TAZ knockout decreases the abundance of epidermal caveolae in embryos of zebrafish [97]. Recently, endothelial YAP was identified to be crucial in many physiological and pathophysiological processes, including angiogenesis, hypertension, and atherosclerosis [96, 99, 100]. Unidirectional shear stress (USS) increased, while disturbed flow decreased, YAP phosphorylation (Ser127) in ECs. Consistently, YAP phosphorylation (Ser127) in the aortic arch was higher than that in the thoracic aorta [101].
Overexpression of the $\beta 3$ mutation (with cytoplasmic domain deletion) abolished USS-induced YAP phosphorylation (Ser127). Knockdown of $\beta 3$ reversed the effects of the RGD-containing peptide (GRGDSP) and $\mathrm{MnCl}_{2}$ (a putative integrin agonist) on YAP phosphorylation and endothelial activation [101]. OSS led to tyrosine phosphorylation of YAP (Tyr357) and strong, continuous nuclear translocation of YAP in ECs, which is dependent on $\alpha 5 \beta 1$ activation [80, 102]. Pretreatment with ATN-161, an $\alpha 5$ blocking peptide, did not alter the subcellular localization of YAP in HUVECs under static conditions, but completely blocked the OSS-induced YAP nuclear translocation [80]. Consistently, the nuclear localization of YAP in the inner curvature of the aortic arch, but not the outer curvature of the aortic arch or the thoracic aorta of wild-type mice, was abolished in $\alpha 5$ heterozygous knockout mice [80]. Tie2Cremediated YAP overexpression mice showed marked induction of retinal angiogenesis during development, while endothelial knockout of YAP leads to embryonic death in mice $[103,104]$. EC-specific YAP knockdown via adenoassociated viruses (AAV) expressing CA-YAP/TAZ reduced plaque formation in $\mathrm{ApoE}^{-/-}$mice [101]. In contrast, Tie2Cre-mediated YAP overexpression aggravated both

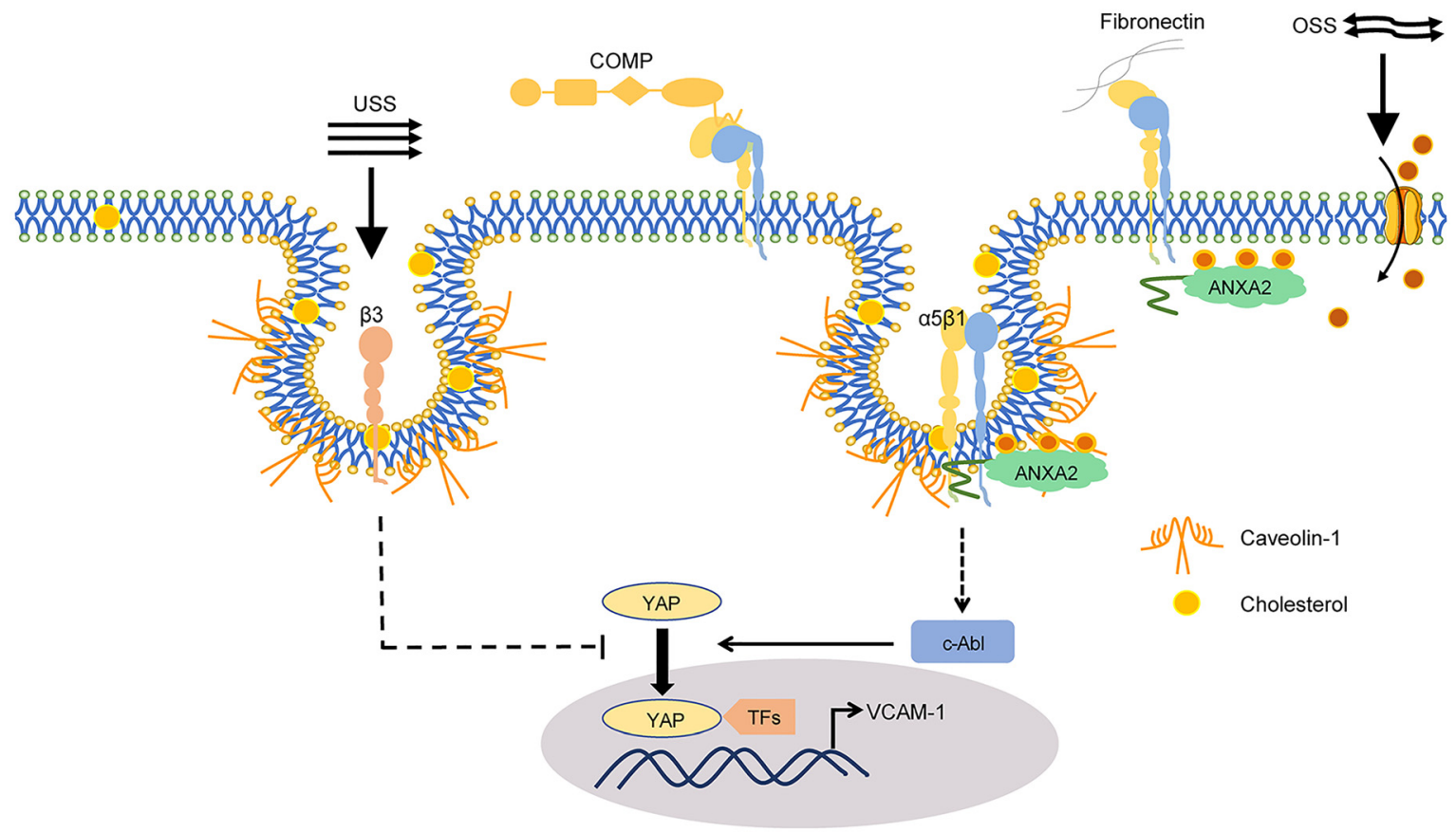

Figure 5: Endothelial caveolae, shear stress, and integrin-YAP. ANXA2 binds with $\alpha 5$ by undergoing a conformational change in ECs under 0 SS and promotes $\alpha 5$ translocation to caveolae. COMP directly interacts with $\alpha 5$ and inhibits both disturbed flow-induced and fibronectin-induced $\alpha 5 \beta 1$ activation by inducing a more bent conformation of $\alpha 5 \beta 1$. Phosphorylation of c-Abl induced by $\alpha 5 \beta 1$ activation induces tyrosine phosphorylation of YAP and promotes its nuclear translocation. In contrast, USS enhances serine phosphorylation of YAP and blunts its nuclear translocation via activating $\beta 3$. Nuclear YAP binds with transcription factors and promotes the expression of inflammatory genes such as VCAM-1. ANXA2: Annexin A2; COMP: Cartilage oligomeric matrix protein; OSS: Oscillatory shear stress; TFs: transcription factors; USS: unidirectional shear stress; VCAM-1: Vascular cell adhesion molecule-1; YAP: Yes-associated protein. 
Western diet-induced and partial ligation-induced plaque formation in $\mathrm{ApoE}^{-/-}$mice and blunted the atheroprotective effects of ATN-161 [80].

\section{Perspectives and conclusion}

Since the identification of caveolae in the 1950s, numerous studies have demonstrated that they affect various physiological and pathophysiological processes. In this review, we discuss several signaling pathways, potentially related to hypertension and atherosclerosis, influenced by caveolae in ECs. Since these two are chronic disorders orchestrated by both local and systemic factors, and caveolin-1 secretion occurs in some types of cells, further studies focusing on caveolae function in vascular and immune cells will provide a more precise assessment of caveolae/caveolin in the progression of diseases. The endothelial lining of blood vessels shows remarkable spatial and temporal heterogeneity in both structure and function. Moreover, the density and components of endothelial caveolae differ in tissues and conditions. Hence, high-throughput single-cell analysis might facilitate addressing these issues and complement our understanding of endothelial caveolae and their downstream signaling pathways. Although studies have revealed the convincing and diversified roles of endothelial caveolae in signal transduction, further investigations on the mechanism of precise regulation of signaling and therapeutic strategies targeting endothelial caveolae-related molecules in cardiovascular diseases are warranted.

Research funding: This work was supported by National Key Research and Development Program of China Grant (2019YFA0802003), National Natural Science Foundation of China Grants (81730014, 81790621, 82070451, and 81870313), Young Elite Scientists Sponsorship Program by CAST (2019QNRC001), and Tianjin Municipal Science and Technology Project (18JCZDJC44900 and 17JCJQJC45700). Dr. Yi Zhu is a fellow in the Jiangsu Collaborative Innovation Center for Cardiovascular Disease Translational Medicine.

Author contributions: J.H. and Z.C. drafted the manuscript and designed figures. Y.Z. supervised the work and made critical revisions to the manuscript. All authors read and approved the manuscript.

Competing interests: None.

Informed consent: Not applicable.

Ethical approval: Not applicable.

\section{References}

1. Parton RG. Caveolae: structure, function, and relationship to disease. Annu Rev Cell Dev Biol 2018;34:111-36.

2. Pelkmans L, Zerial $M$. Kinase-regulated quantal assemblies and kiss-and-run recycling of caveolae. Nature 2005;436:128-33.

3. Fra AM, Williamson E, Simons K, Parton RG. De novo formation of caveolae in lymphocytes by expression of VIP21-caveolin. Proc Natl Acad Sci U S A 1995;92:8655-9.

4. Stan RV. Structure and function of endothelial caveolae. Microsc Res Tech 2002;57:350-64.

5. Schnitzer JE, Carley WW, Palade GE. Specific albumin binding to microvascular endothelium in culture. Am J Physiol 1988;254: H425-37.

6. Rizzo V, Morton C, DePaola N, Schnitzer JE, Davies PF. Recruitment of endothelial caveolae into mechanotransduction pathways by flow conditioning in vitro. Am J Physiol Heart Circ Physiol 2003;285:H1720-9.

7. Frank PG, Lee H, Park DS, Tandon NN, Scherer PE, Lisanti MP. Genetic ablation of caveolin-1 confers protection against atherosclerosis. Arterioscler Thromb Vasc Biol 2004;24:98-105.

8. Fernández-Hernando C, Yu J, Suárez Y, Rahner C, Dávalos A, Lasunción MA, et al. Genetic evidence supporting a critical role of endothelial caveolin-1 during the progression of atherosclerosis. Cell Metab 2009;10:48-54.

9. Potje SR, Grando MD, Chignalia AZ, Antoniali C, Bendhack LM. Reduced caveolae density in arteries of SHR contributes to endothelial dysfunction and ROS production. Sci Rep 2019;9:6696.

10. Czikora I, Feher A, Lucas R, Fulton DJ, Bagi Z. Caveolin-1 prevents sustained angiotensin II-induced resistance artery constriction and obesity-induced high blood pressure. Am J Physiol Heart Circ Physiol 2015;308:H376-85.

11. Ushio-Fukai M, Alexander RW. Caveolin-dependent angiotensin II type 1 receptor signaling in vascular smooth muscle. Hypertension 2006;48:797-803.

12. Drab M, Verkade P, Elger M, Kasper M, Lohn M, Lauterbach B, et al. Loss of caveolae, vascular dysfunction, and pulmonary defects in caveolin-1 gene-disrupted mice. Science 2001;293: 2449-52.

13. Haas AV, Baudrand R, Easly RM, Murray GR, Touyz RM, Pojoga LH, et al. Interplay between statins, Cav1 (Caveolin-1), and aldosterone. Hypertension 2020;76:962-7.

14. Asterholm IW, Mundy DI, Weng J, Anderson RG, Scherer PE. Altered mitochondrial function and metabolic inflexibility associated with loss of caveolin-1. Cell Metab 2012;15:171-85.

15. Bosch M, Mari M, Herms A, Fernandez A, Fajardo A, Kassan A, et al. Caveolin-1 deficiency causes cholesterol-dependent mitochondrial dysfunction and apoptotic susceptibility. Curr Biol 2011;21:681-6.

16. Gu MX, Fu Y, Sun XL, Ding YZ, Li CH, Pang W, et al. Proteomic analysis of endothelial lipid rafts reveals a novel role of statins in antioxidation. J Proteome Res 2012;11:2365-73.

17. Tian J, Popal MS, Huang R, Zhang M, Zhao X, Song X. Caveolin as a novel potential therapeutic target in cardiac and vascular diseases: a mini review. Aging Dis 2020;11:378-89. 
18. Ference BA, Ginsberg HN, Graham I, Ray KK, Packard CJ, Bruckert $E$, et al. Low-density lipoproteins cause atherosclerotic cardiovascular disease. 1. Evidence from genetic, epidemiologic, and clinical studies. A consensus statement from the European Atherosclerosis Society Consensus Panel. Eur Heart J 2017;38: 2459-72.

19. Qin L, Zhu N, Ao BX, Liu C, Shi YN, Du K, et al. Caveolae and caveolin-1 integrate reverse cholesterol transport and inflammation in atherosclerosis. Int J Mol Sci 2016;17:429.

20. Zhang X, Sessa WC, Fernandez-Hernando C. Endothelial transcytosis of lipoproteins in atherosclerosis. Front Cardiovasc Med 2018;5:130.

21. Gerbod-Giannone MC, Dallet L, Naudin G, Sahin A, Decossas M, Poussard S, et al. Involvement of caveolin-1 and CD36 in native LDL endocytosis by endothelial cells. Biochim Biophys Acta Gen Subj 2019;1863:830-8.

22. Zhu Y, Liao HL, Wang N, Yuan Y, Ma KS, Verna L, et al. Lipoprotein promotes caveolin-1 and Ras translocation to caveolae: role of cholesterol in endothelial signaling. Arterioscler Thromb Vasc Biol 2000;20:2465-70.

23. Murata M, Peranen J, Schreiner R, Wieland F, Kurzchalia TV, Simons K. VIP21/caveolin is a cholesterol-binding protein. Proc Natl Acad Sci U S A 1995;92:10339-43.

24. Liao H, Langmann T, Schmitz G, Zhu Y. Native LDL upregulation of ATP-binding cassette transporter-1 in human vascular endothelial cells. Arterioscler Thromb Vasc Biol 2002;22:127-32.

25. Costet $P$, Luo $Y$, Wang N, Tall AR. Sterol-dependent transactivation of the $A B C 1$ promoter by the liver $X$ receptor/ retinoid X receptor. J Biol Chem 2000;275:28240-5.

26. Zhu M, Fu Y, Hou Y, Wang N, Guan Y, Tang C, et al. Laminar shear stress regulates liver $X$ receptor in vascular endothelial cells. Arterioscler Thromb Vasc Biol 2008;28:527-33.

27. Meurs I, Lammers B, Zhao Y, Out R, Hildebrand RB, Hoekstra M, et al. The effect of $A B C G 1$ deficiency on atherosclerotic lesion development in LDL receptor knockout mice depends on the stage of atherogenesis. Atherosclerosis 2012;221:41-7.

28. Whetzel AM, Sturek JM, Nagelin MH, Bolick DT, Gebre AK, Parks JS, et al. ABCG1 deficiency in mice promotes endothelial activation and monocyte-endothelial interactions. Arterioscler Thromb Vasc Biol 2010;30:809-17.

29. Munch G, Bultmann A, Li Z, Holthoff HP, Ullrich J, Wagner S, et al. Overexpression of $A B C G 1$ protein attenuates arteriosclerosis and endothelial dysfunction in atherosclerotic rabbits. Heart Int 2012; 7:e12.

30. Xue S, Wang J, Zhang X, Shi Y, Li B, Bao Q, et al. Endothelial ATP-binding cassette $\mathrm{G} 1$ in mouse endothelium protects against hemodynamic-induced atherosclerosis. Biochem Biophys Res Commun 2016;477:247-54.

31. Sampietro T, Tuoni M, Ferdeghini M, Ciardi A, Marraccini P, Prontera $\mathrm{C}$, et al. Plasma cholesterol regulates soluble cell adhesion molecule expression in familial hypercholesterolemia. Circulation 1997;96:1381-5.

32. Millán J, Hewlett L, Glyn M, Toomre D, Clark P, Ridley AJ. Lymphocyte transcellular migration occurs through recruitment of endothelial ICAM-1 to caveola- and F-actin-rich domains. Nat Cell Biol 2006;8:113-23.

33. Fu C, He J, Li C, Shyy JY, Zhu Y. Cholesterol increases adhesion of monocytes to endothelium by moving adhesion molecules out of caveolae. Biochim Biophys Acta 2010;1801:702-10.
34. Kawashima S, Yokoyama M. Dysfunction of endothelial nitric oxide synthase and atherosclerosis. Arterioscler Thromb Vasc Biol 2004;24:998-1005.

35. García-Cardeña G, Martasek P, Masters BS, Skidd PM, Couet J, Li $S$, et al. Dissecting the interaction between nitric oxide synthase (NOS) and caveolin. Functional significance of the nos caveolin binding domain in vivo. J Biol Chem 1997;272:25437-40.

36. Sun X, Lv H, Zhao P, He J, Cui Q, Wei M, et al. Commutative regulation between endothelial NO synthase and insulin receptor substrate 2 by microRNAs. J Mol Cell Biol 2019;11:509-20.

37. Knowles JW, Reddick RL, Jennette JC, Shesely EG, Smithies O, Maeda N. Enhanced atherosclerosis and kidney dysfunction in eNOS(-/-)Apoe(-/-) mice are ameliorated by enalapril treatment. J Clin Invest 2000;105:451-8.

38. Sharma A, Sellers S, Stefanovic N, Leung C, Tan SM, Huet O, et al. Direct endothelial nitric oxide synthase activation provides atheroprotection in diabetes-accelerated atherosclerosis. Diabetes 2015;64:3937.

39. Kuhlencordt PJ, Gyurko R, Han F, Scherrer-Crosbie M, Aretz TH, Hajjar R, et al. Accelerated atherosclerosis, aortic aneurysm formation, and ischemic heart disease in apolipoprotein E/endothelial nitric oxide synthase double-knockout mice. Circulation 2001;104:448-54.

40. Ozaki M, Kawashima S, Yamashita T, Hirase T, Namiki M, Inoue N, et al. Overexpression of endothelial nitric oxide synthase accelerates atherosclerotic lesion formation in apoE-deficient mice. J Clin Invest 2002;110:331-40.

41. Al-Brakati AY, Kamishima T, Dart C, Quayle JM. Caveolar disruption causes contraction of rat femoral arteries via reduced basal NO release and subsequent closure of BKCa channels. Peer) 2015;3:e966.

42. Xu Y, Henning RH, van der Want JJ, van Buiten A, van Gilst WH, Buikema H. Disruption of endothelial caveolae is associated with impairment of both NO- as well as EDHF in acetylcholine-induced relaxation depending on their relative contribution in different vascular beds. Life Sci 2007;80:1678-85.

43. Gratton JP, Fontana J, O'Connor DS, Garcia-Cardena G, McCabe TJ, Sessa WC. Reconstitution of an endothelial nitric-oxide synthase (eNOS), hsp90, and caveolin-1 complex in vitro. Evidence that hsp90 facilitates calmodulin stimulated displacement of eNOS from caveolin-1. J Biol Chem 2000;275:22268-72.

44. Michel JB, Feron O, Sase K, Prabhakar P, Michel T. Caveolin versus calmodulin. Counterbalancing allosteric modulators of endothelial nitric oxide synthase. J Biol Chem 1997;272:25907-12.

45. Wen L, Chen Z, Zhang F, Cui X, Sun W, Geary GG, et al. $\mathrm{Ca}^{2+} /$ calmodulin-dependent protein kinase kinase $\beta$ phosphorylation of Sirtuin 1 in endothelium is atheroprotective. Proc Natl Acad Sci U S A 2013;110:E2420-7.

46. Ramírez CM, Zhang X, Bandyopadhyay C, Rotllan N, Sugiyama $M G$, Aryal $B$, et al. Caveolin-1 regulates atherogenesis by attenuating low-density lipoprotein transcytosis and vascular inflammation independently of endothelial nitric oxide synthase activation. Circulation 2019;140:225-39.

47. Boopathy GTK, Kulkarni M, Ho SY, Boey A, Chua EWM, Barathi VA, et al. Cavin-2 regulates the activity and stability of endothelial nitric-oxide synthase (eNOS) in angiogenesis. J Biol Chem 2017; 292:17760-76.

48. Zhu Y, Liao HL, Niu XL, Yuan Y, Lin T, Verna L, et al. Low density lipoprotein induces eNOS translocation to membrane caveolae: 
the role of RhoA activation and stress fiber formation. Biochim Biophys Acta 2003;1635:117-26.

49. Mineo C, Shaul PW. Regulation of eNOS in caveolae. Adv Exp Med Biol 2012;729:51-62.

50. Shaul PW. Endothelial nitric oxide synthase, caveolae and the development of atherosclerosis. J Physiol 2003;547:21-33.

51. Gerhardt T, Ley K. Monocyte trafficking across the vessel wall. Cardiovasc Res 2015;107:321-30.

52. Suo J, Ferrara DE, Sorescu D, Guldberg RE, Taylor WR, Giddens DP. Hemodynamic shear stresses in mouse aortas: implications for atherogenesis. Arterioscler Thromb Vasc Biol 2007;27: 346-51.

53. Niu N, Xu S, Xu Y, Little PJ, Jin ZG. Targeting mechanosensitive transcription factors in atherosclerosis. Trends Pharmacol Sci 2019;40:253-66.

54. Tzima E, Del Pozo MA, Kiosses WB, Mohamed SA, Li S, Chien S, et al. Activation of Rac1 by shear stress in endothelial cells mediates both cytoskeletal reorganization and effects on gene expression. EMBO J 2002;21:6791-800.

55. Parton RG, del Pozo MA. Caveolae as plasma membrane sensors, protectors and organizers. Nat Rev Mol Cell Biol 2013;14:98-112.

56. Boyer PD. The ATP synthase-a splendid molecular machine. Annu Rev Biochem 1997;66:717-49.

57. Walker JE, Fearnley IM, Gay NJ, Gibson BW, Northrop FD, Powell SJ, et al. Primary structure and subunit stoichiometry of F1-ATPase from bovine mitochondria. J Mol Biol 1985;184:677-701.

58. Chin RM, Fu X, Pai MY, Vergnes L, Hwang H, Deng G, et al. The metabolite alpha-ketoglutarate extends lifespan by inhibiting ATP synthase and TOR. Nature 2014;510:397-401.

59. Terni B, Boada J, Portero-Otin M, Pamplona R, Ferrer I. Mitochondrial ATP-synthase in the entorhinal cortex is a target of oxidative stress at stages I/II of Alzheimer's disease pathology. Brain Pathol 2010;20:222-33.

60. Das B, Mondragon MO, Sadeghian M, Hatcher VB, Norin AJ. A novel ligand in lymphocyte-mediated cytotoxicity: expression of the beta subunit of $\mathrm{H}+$ transporting ATP synthase on the surface of tumor cell lines. J Exp Med 1994;180:273-81.

61. Moser TL, Stack MS, Asplin I, Enghild JJ, Hojrup P, Everitt L, et al. Angiostatin binds ATP synthase on the surface of human endothelial cells. Proc Natl Acad Sci USA 1999;96:2811-6.

62. Veitonmäki N, Cao R, Wu LH, Moser TL, Li B, Pizzo SV, et al. Endothelial cell surface ATP synthase-triggered caspaseapoptotic pathway is essential for k1-5-induced antiangiogenesis. Canc Res 2004;64:3679-86.

63. Radojkovic C, Genoux A, Pons V, Combes G, de Jonge H, Champagne $E$, et al. Stimulation of cell surface F1-ATPase activity by apolipoprotein $\mathrm{A}-\mathrm{I}$ inhibits endothelial cell apoptosis and promotes proliferation. Arterioscler Thromb Vasc Biol 2009;29:1125-30.

64. Wang T, Chen Z, Wang X, Shyy JY, Zhu Y. Cholesterol loading increases the translocation of ATP synthase beta chain into membrane caveolae in vascular endothelial cells. Biochim Biophys Acta 2006;1761:1182-90.

65. Yamamoto K, Shimizu N, Obi S, Kumagaya S, Taketani Y, Kamiya A, et al. Involvement of cell surface ATP synthase in flow-induced ATP release by vascular endothelial cells. Am J Physiol Heart Circ Physiol 2007;293:H1646-53.

66. Fu Y, Hou Y, Fu C, Gu M, Li C, Kong W, et al. A novel mechanism of gamma/delta T-lymphocyte and endothelial activation by shear stress: the role of ecto-ATP synthase beta chain. Circ Res 2011; 108:410-7.
67. Yurdagul A, Jr, Orr AW. Blood brothers: hemodynamics and cellmatrix interactions in endothelial function. Antioxid Redox Signal 2016;25:415-34.

68. Finney AC, Stokes KY, Pattillo CB, Orr AW. Integrin signaling in atherosclerosis. Cell Mol Life Sci 2017;74:2263-82.

69. Chen J, Green J, Yurdagul A Jr, Albert P, McInnis MC, Orr AW. $\alpha v \beta 3$ integrins mediate flow-induced NF-KB activation, proinflammatory gene expression, and early atherogenic inflammation. Am J Pathol 2015;185:2575-89.

70. Yurdagul A, Jr, Green J, Albert P, McInnis MC, Mazar AP, Orr AW. $\alpha 5 \beta 1$ integrin signaling mediates oxidized low-density lipoprotein-induced inflammation and early atherosclerosis. Arterioscler Thromb Vasc Biol 2014;34:1362-73.

71. Sun X, Fu Y, Gu M, Zhang L, Li D, Li H, et al. Activation of integrin $\alpha 5$ mediated by flow requires its translocation to membrane lipid rafts in vascular endothelial cells. Proc Natl Acad Sci U S A 2016; 113:769-74.

72. Yun S, Budatha M, Dahlman JE, Coon BG, Cameron RT, Langer R, et al. Interaction between integrin $\alpha 5$ and PDE4D regulates endothelial inflammatory signalling. Nat Cell Biol 2016;18:1043-53.

73. Budatha M, Zhang J, Zhuang ZW, Yun S, Dahlman JE, Anderson $D G$, et al. Inhibiting integrin $\alpha 5$ cytoplasmic domain signaling reduces atherosclerosis and promotes arteriogenesis. J Am Heart Assoc 2018;7:e007501.

74. Izawa Y, Gu YH, Osada T, Kanazawa M, Hawkins BT, Koziol JA, et al. $\beta 1$-integrin-matrix interactions modulate cerebral microvessel endothelial cell tight junction expression and permeability. J Cerebr Blood Flow Metab 2018;38:641-58.

75. Pulous FE, Grimsley-Myers CM, Kansal S, Kowalczyk AP, Petrich BG. Talin-dependent integrin activation regulates VE-cadherin localization and endothelial cell barrier function. Circ Res 2019; 124:891-903.

76. Yun S, Hu R, Schwaemmle ME, Scherer AN, Zhuang Z, Koleske AJ, et al. Integrin $\alpha 5 \beta 1$ regulates PP2A complex assembly through PDE4D in atherosclerosis. J Clin Invest 2019;129:4863-74.

77. del Pozo MA, Balasubramanian N, Alderson NB, Kiosses WB, Grande-García A, Anderson RG, et al. Phospho-caveolin-1 mediates integrin-regulated membrane domain internalization. Nat Cell Biol 2005;7:901-8.

78. del Pozo MA, Alderson NB, Kiosses WB, Chiang HH, Anderson RG, Schwartz MA. Integrins regulate Rac targeting by internalization of membrane domains. Science 2004;303:839-42.

79. Du J, Chen X, Liang X, Zhang G, Xu J, He L, et al. Integrin activation and internalization on soft ECM as a mechanism of induction of stem cell differentiation by ECM elasticity. Proc Natl Acad Sci U S A 2011;108:9466-71.

80. Li B, He J, Lv H, Liu Y, Lv X, Zhang C, et al. C-Abl regulates YAPY357 phosphorylation to activate endothelial atherogenic responses to disturbed flow. J Clin Invest 2019;129:1167-79.

81. Lv H, Wang H, Quan M, Zhang C, Fu Y, Zhang L, et al. Cartilage oligomeric matrix protein fine-tunes disturbed flow-induced endothelial activation and atherogenesis. Matrix Biol 2021;95:32-51.

82. Riessen R, Fenchel M, Chen H, Axel DI, Karsch KR, Lawler J. Cartilage oligomeric matrix protein (thrombospondin-5) is expressed by human vascular smooth muscle cells. Arterioscler Thromb Vasc Biol 2001;21:47-54.

83. Fu Y, Gao C, Liang Y, Wang M, Huang Y, Ma W, et al. Shift of macrophage phenotype due to cartilage oligomeric matrix protein deficiency drives atherosclerotic calcification. Circ Res 2016;119:261-76. 
84. Wang L, Zheng J, Du Y, Huang Y, Li J, Liu B, et al. Cartilage oligomeric matrix protein maintains the contractile phenotype of vascular smooth muscle cells by interacting with alpha(7)beta(1) integrin. Circ Res 2010;106:514-25.

85. Radel C, Carlile-Klusacek M, Rizzo V. Participation of caveolae in beta1 integrin-mediated mechanotransduction. Biochem Biophys Res Commun 2007;358:626-31.

86. Sohn J, Lin H, Fritch MR, Tuan RS. Influence of cholesterol/ caveolin-1/caveolae homeostasis on membrane properties and substrate adhesion characteristics of adult human mesenchymal stem cells. Stem Cell Res Ther 2018;9:86.

87. Jung SH, Lee M, Park HA, Lee HC, Kang D, Hwang HJ, et al. Integrin $\alpha 6 \beta 4-S r c-A K T$ signaling induces cellular senescence by counteracting apoptosis in irradiated tumor cells and tissues. Cell Death Differ 2019;26:245-59.

88. Gerke V, Creutz CE, Moss SE. Annexins: linking $\mathrm{Ca}^{2+}$ signalling to membrane dynamics. Nat Rev Mol Cell Biol 2005;6:449-61.

89. Yang SF, Hsu HL, Chao TK, Hsiao CJ, Lin YF, Cheng CW. Annexin A2 in renal cell carcinoma: expression, function, and prognostic significance. Urol Oncol 2015;33:22.e11-22.e21.

90. Wang CY, Lin CF. Annexin A2: its molecular regulation and cellular expression in cancer development. Dis Markers 2014;2014:308976.

91. Caron D, Boutchueng-Djidjou M, Tanguay RM, Faure RL. Annexin A2 is SUMOylated on its N-terminal domain: regulation by insulin. FEBS Lett 2015;589:985-91.

92. Liao L, Zheng B, Yi B, Liu C, Chen L, Zeng Z, et al. Annexin A2-modulated proliferation of pulmonary arterial smooth muscle cells depends on caveolae and caveolin-1 in hepatopulmonary syndrome. Exp Cell Res 2017;359:266-74.

93. Staquicini DI, Rangel R, Guzman-Rojas L, Staquicini FI, Dobroff AS, Tarleton CA, et al. Intracellular targeting of annexin A2 inhibits tumor cell adhesion, migration, and in vivo grafting. Sci Rep 2017;7:4243.

94. Zhang C, Zhou T, Chen Z, Yan M, Li B, Lv H, et al. Coupling of integrin $\alpha 5$ to annexin $A 2$ by flow drives endothelial activation. Circ Res 2020;127:1074-90.
95. Mitra SK, Hanson DA, Schlaepfer DD. Focal adhesion kinase: in command and control of cell motility. Nat Rev Mol Cell Biol 2005; 6:56-68.

96. Yu FX, Zhao B, Guan KL. Hippo pathway in organ size control, tissue homeostasis, and cancer. Cell 2015;163:811-28.

97. Rausch V, Bostrom JR, Park J, Bravo IR, Feng Y, Hay DC, et al. The Hippo pathway regulates caveolae expression and mediates flow response via caveolae. Curr Biol 2019;29: 242-55.e6.

98. Strippoli R, Sandoval P, Moreno-Vicente R, Rossi L, Battistelli C, Terri $\mathrm{M}$, et al. Caveolin1 and YAP drive mechanically induced mesothelial to mesenchymal transition and fibrosis. Cell Death Dis 2020;11:647.

99. He J, Bao Q, Yan M, Liang J, Zhu Y, Wang C, et al. The role of Hippo/yes-associated protein signalling in vascular remodelling associated with cardiovascular disease. $\mathrm{Br}$ J Pharmacol 2018;175:1354-61.

100. Yao L, He J, Li B, Yan M, Wang H, Tan L, et al. Regulation of YAP by mammalian target of Rapamycin complex 1 in endothelial cells controls blood pressure through COX-2/mPGES-1/PGE(2) cascade. Hypertension 2019;74:936-46.

101. Wang L, Luo JY, Li B, Tian XY, Chen LJ, Huang Y, et al. IntegrinYAP/TAZ-JNK cascade mediates atheroprotective effect of unidirectional shear flow. Nature 2016;540:579-82.

102. Yang $\mathrm{Y}, \mathrm{Ma} \mathrm{Q}$, Li Z, Wang H, Zhang C, Liu Y, et al. Harmine alleviates atherogenesis by inhibiting disturbed flow-mediated endothelial activation via protein tyrosine phosphatase PTPN14 and YAP. Br J Pharmacol 2021;178:1524-40.

103. Zhang H, von Gise A, Liu Q, Hu T, Tian X, He L, et al. Yap1 is required for endothelial to mesenchymal transition of the atrioventricular cushion. J Biol Chem 2014;289: 18681-92.

104. He J, Bao Q, Zhang Y, Liu M, Lv H, Liu Y, et al. Yes-associated protein promotes angiogenesis via signal transducer and activator of transcription 3 in endothelial cells. Circ Res 2018; 122:591-605. 